Research Article

\title{
Computing the Permanent of the Laplacian Matrices of Nonbipartite Graphs
}

\author{
Xiaoxue Hu and Grace Kalaso \\ School of Science, Zhejiang University of Science and Technology, Hangzhou 310023, China \\ Correspondence should be addressed to Xiaoxue Hu; xxhu@zjnu.edu.cn
}

Received 31 December 2020; Accepted 12 June 2021; Published 23 June 2021

Academic Editor: Francisco Balibrea

Copyright (C) 2021 Xiaoxue $\mathrm{Hu}$ and Grace Kalaso. This is an open access article distributed under the Creative Commons Attribution License, which permits unrestricted use, distribution, and reproduction in any medium, provided the original work is properly cited.

Let $G$ be a graph with Laplacian matrix $L(G)$. Denote by per $L(G)$ the permanent of $L(G)$. In this study, we investigate the problem of computing the permanent of the Laplacian matrix of nonbipartite graphs. We show that the permanent of the Laplacian matrix of some classes of nonbipartite graphs can be formulated as the composite of the determinants of two matrices related to those Laplacian matrices. In addition, some recursion formulas on per $L(G)$ are deduced.

\section{Introduction}

All graphs in this paper are restricted to be simple. Let $G=$ $(V(G), E(G))$ be a connected simple graph with vertex set $V(G)=\{1,2, \ldots, n\}$ and edge set $E(G)$. Denoted by $d_{G}(v)$, or short for $d_{v}$ if there is no confusion, the degree of $v \in V(G)$. Let $G$ be a graph of order $n$. The adjacency matrix $A=A(G)=\left[a_{i j}\right]_{n \times n}$ related to $G$ is defined as $a_{i j}=1$ if and only if $i$ and $j$ are adjacent and 0 otherwise. Let $D=D(G)$ be the diagonal matrix of the graph $G$ whose $(i, i)$ entry is $d_{i}, i=1,2, \ldots, n$. The Laplacian matrix related to $G$ is defined by $L(G)=D(G)-A(G)$, which has been extensively investigated for a long time. For more properties of the Laplacian matrix of graphs, reader may refer to the books $[1,2]$, the surveys [3-5], and the references therein.

The determinant and permanent of an $n \times n$ square matrix $A=\left[a_{i j}\right]_{n \times n}$ is defined by

$$
\begin{aligned}
& \operatorname{det} A=\sum \operatorname{sgn}(\sigma) \prod_{i=1}^{n} a_{i, \sigma(i)}, \\
& \operatorname{per} A=\sum \prod_{i=1}^{n} a_{i, \sigma(i)},
\end{aligned}
$$

respectively, where the summation extends over all permutations $\sigma$ of $\{1,2, \ldots, n\}$ and $\operatorname{sgn}(\sigma)=1$ if $\sigma$ is the product of an even number of transpositions and $\operatorname{sgn}(\sigma)=-1$ otherwise.

The formula for permanent is similar to, even simpler than, the formula for determinant. However, there is a polynomial algorithm for calculating determinants, whereas calculating permanents is $\# P$-complete as shown by Valiant [6]. Therefore, it is reasonable to ask if perhaps computing the permanent of a matrix can be somehow converted to computing the determinant of a related matrix. Readers may refer to [7-9] and the references therein for more information on this question.

There are many results on the permanent, or the permanent polynomial per $(I \lambda-A)$, in terms of the adjacency matrix of bipartite graphs, see, for example, [10, 11], whereas, there are few formulaic results on the permanent of the matrix related to the nonbipartite graphs.

Merris et al. [12] first studied the permanent of the Laplacian matrix, in which the polynomial per $(x I-L(G))$ is suggested to distinguish nonisomorphic trees, and lower bounds on the permanent of $L(G)$, per $L(G)$, were conjectured. After that, several lower bounds on per $L(G)$ were proved by Brualdi and Goldwasser [13] and Merris [11]. For more results on the permanent of the Laplacian matrix, we refer the reader to [11-15] and the references contained therein. 
Pólya's permanent problem is well known, which has many equivalent versions, such as, given a matrix $A$, it was possible to change the signs of some of the entries of $A$ to give a new matrix $B$ such that the corresponding terms of per $A$ and $\operatorname{det} B$ were equal. Pólya's permanent problem still remains open. We refer the readers to the survey [16] for the history and different versions of Pólya's permanent problem.

In general, for Pólya's permanent problem, we only consider exactly one objective matrix, that is, for a given matrix $A$, we want to find one objective matrix $B$ related to $A$ such that per $A=\operatorname{det} B$. Naturally, the following question, which can be considered as a generalized version Pólya's permanent problem, is interesting.

For a given matrix $A$, can we find two matrices $B$ and $C$ related to $A$ such that $\operatorname{per} A$ is the composite of $\operatorname{det} B$ and $\operatorname{det} C$ ?

We, in this paper, continue to investigate the problem of computing the permanent of the Laplacian matrix of graphs. In Section 2, we give some preliminary results, including the combinatorial description of per $L\left(G^{\sigma}\right)$ and $\operatorname{per} L^{\mathbf{i}}(G)$ in terms of Sachs subgraphs, where $G^{\sigma}$ is an oriented graph defined in below and $L^{\mathbf{i}}(G)=D(G)-A(G) \mathbf{i}$ with $\mathbf{i}^{2}=-1$. In Section 3, we first deduce a formula on the permanent of the Laplacian matrix of a class of bipartite graphs. Then, we show that the permanent of the Laplacian matrix of a class of nonbipartite graphs can be formulated as the composite of the determinants of two matrices related to their Laplacian matrices. In addition, some recursion formulas on per $L(G)$ are obtained, which can simplify the calculation on the permanent of the Laplacian matrix of more general classes of nonbipartite graphs.

\section{Preliminary}

Let $G$ be a connected graph with vertex set $V$ and edge set $E$. An edge $e \in E$ is called a bridge if the resultant graph obtained from $G$ by deleting the edge $e$ has two components. For a nonempty subset $W$ of $V(G)$, the subgraph with vertex set $W$ and edge set consisting of those pairs of vertices that are edges in $G$ is called the induced subgraph of $G$, denoted by $G[W]$. Denote by $G \backslash U$, where $U \subseteq V$, and the graph is obtained from $G$ by removing the vertices of $U$ together with all edges incident to them. Let $L^{\mathbf{i}}(G)=D(G)-A(G) \mathbf{i}$, where $D(G)$ and $A(G)$ are defined as above and $\mathbf{i}^{2}=-1$.

Let $G$ be a graph with vertex set $V=\{1,2, \ldots, n\}$. The matrix $M=\left[m_{i j}\right]_{n \times n}$ is called a graphical matrix of $G$ if $m_{i j} \neq 0, i \neq j$, if and only if $(i, j)$ is an edge of $G$. Then, the adjacency matrix $A(G)$, the Laplacian matrix $L(G)$, and the matrix $L^{\mathbf{i}}(G)$ are all graphical matrices with respect to the given graph $G$. Let $G$ be a graph and $M$ be a graphical matrix of $G$. Suppose that $e=(i, j)$ is an edge of $G$ and $G_{1}$ is a subgraph of $G$. Denote by $M[G \backslash e]$ the matrix obtained from $M$ by replacing the entries $m_{i j}$ and $m_{j i}$ by zeros, and by $M\left[G_{1}\right]$ the principle submatrix of $M$ corresponding to the subgraph $G_{1}$. Then, $M[G \backslash e]$ (resp. $M\left[G_{1}\right]$ ) is a graphical matrix of $G \backslash e$ (resp. $G_{1}$ ) if and only if $M$ is a graphical matrix of $G$.
For an undirected graph $G$, the subgraph $H$ of $G$ is called a Sachs subgraph of $G$ if each component of $H$ is either a single edge or a cycle; see, for instance, [16]. For a Sachs subgraph $H$, denote, by $c_{o}(H)$ and $c(H)$, the number of odd cycles and cycles contained in $H$, respectively.

An oriented graph $G^{\sigma}$ is a graph obtained from an undirected graph $G$ by orienting each edge of $G$ a direction. Then, $G$ is referred as the underlying graph of $G^{\sigma}$. We should point out that our oriented graph considered its underlying graph in terms of defining matching, degree, path, and connectedness. An even oriented cycle $C$ in $G^{\sigma}$ is called oddly oriented (resp. evenly oriented) if, for either choice of direction of traversal around $C$, the number of edges of $C$ directed in the direction of traversal is odd (resp. even). Clearly, this is independent of the initial choice of direction of traversal. An oriented graph $G^{\sigma}$ is Pfaffian if every even oriented cycle of $G$ is oddly oriented in $G^{\sigma}$. A graph is called Pfaffian if such a graph has a Pfaffian orientation, see [16].

Let $G^{\sigma}$ be an oriented graph with vertex set $V\left(G^{\sigma}\right)=$ $\{1,2, \ldots, n\}$. The adjacency matrix $A\left(G^{\sigma}\right)=\left[a_{i j}\right]_{n \times n}$ of $G^{\sigma}$ is defined as $a_{i j}=-a_{j i}=1$ if $i j$ is an edge of $G^{\sigma}$ with tail $i$ and head $j$ and $a_{i j}=0$, otherwise. The Laplacian matrix $L\left(G^{\sigma}\right)$ of $G^{\sigma}$ is defined as $L\left(G^{\sigma}\right)=D\left(G^{\sigma}\right)-A\left(G^{\sigma}\right)$, where $D\left(G^{\sigma}\right)$ is the degree diagonal matrix of its corresponding underlying graph $G$. Obviously, $L\left(G^{\sigma}\right)$ is a graphical matrix of $G^{\sigma}$, as well as of $G$. We refer to $[17,18]$ and the references therein for more spectral properties on the adjacency matrix of oriented graphs.

The subgraph, denoted by $H^{\sigma}$, of a given oriented graph $G^{\sigma}$ is called a Sachs oriented subgraph of $G^{\sigma}$ if each component of $H^{\sigma}$ is either a single edge or a cycle with length even; see examples [18]. For a given Sachs oriented subgraph $H^{\sigma}$, denote by $c^{+}\left(H^{\sigma}\right)$ and $c\left(H^{\sigma}\right)$ the number of evenly even cycles and cycles contained in $H^{\sigma}$, respectively.

For a given oriented graph $G^{\sigma}$, an argument similar to the one given in the proof of Theorem 2.1 in pp. 276-277 of [11] yields the combinatorial description of $\operatorname{det} L\left(G^{\sigma}\right)$; for completeness, we give a simple proof.

Theorem 1. Let $G^{\sigma}$ be a graph of order $n$ and $L\left(G^{\sigma}\right)$ be its Laplacian matrix. Then,

$$
\operatorname{det} L\left(G^{\sigma}\right)=\sum_{K \subseteq\{1,2, \ldots, n\}} \sum_{H^{\sigma} \in H\left(K^{\sigma}\right)}(-1)^{c^{+}\left(H^{\sigma}\right) 2^{c\left(H^{\sigma}\right)}} \prod_{i \notin K} d_{i},
$$

where the first summation is over all induced subgraphs $K^{\sigma}$ of $G^{\sigma}$, the second summation is over all Sachs spanning subgraph $H^{\sigma}$ of $K^{\sigma}$, and $c^{+}\left(H^{\sigma}\right)$ and $c\left(H^{\sigma}\right)$ are defined as above.

Proof. Note that $L\left(G^{\sigma}\right)=D\left(G^{\sigma}\right)-A\left(G^{\sigma}\right)$; then, by the Laplace expansion formula,

$$
\operatorname{det} L\left(G^{\sigma}\right)=\sum_{K \subseteq\{1,2, \ldots, n\}}(-1)^{|K|} \operatorname{det} A[K] \operatorname{det}\left[G^{\sigma} \backslash K\right],
$$

where the summation is over all induced subgraphs $K^{\sigma}$ of $G^{\sigma}$. Similar to the proof in Theorem 2.3 of [18], 


$$
\begin{aligned}
(-1)^{|K|} \operatorname{det} A[K] & =\sum_{H^{\sigma} \in H\left(K^{\sigma}\right)}(-1)^{c^{+}\left(H^{\sigma}\right)} 2^{c\left(H^{\sigma}\right)}, \\
\operatorname{det}\left[G^{\sigma} \backslash K\right] & =\prod_{i \notin K} d_{i},
\end{aligned}
$$

where the summation is over all Sachs spanning subgraph $H^{\sigma}$ of $K^{\sigma}$ and $c^{+}\left(H^{\sigma}\right)$ and $c\left(H^{\sigma}\right)$ are defined as above. Consequently, the proof is complete.

Denote by $\omega(H)$ the number of components contained in $H$. Similarly, we can obtain the following result.

Theorem 2. Let $G$ be a graph of order $n$ and $L^{\mathbf{i}}(G)=D(G)-A(G)$ i. Then,

$$
\operatorname{det} L^{\mathbf{i}}(G)=\sum_{K \subseteq\{1,2, \ldots, n\}}(-\mathbf{i})^{|K|} \sum_{H \in H(K)}(-1)^{\omega(H)} 2^{c(H)} \prod_{i \notin K} d_{i} \text {, }
$$

where the first summation is over all subgraphs $K$ of $G$, the second summation is over all Sach spanning subgraph $H$ of $K$, and $c(H)$ denotes the number of cycles contained in $H$.

\section{The Permanent of the Laplacian Matrix of a Graph}

From the work of Brualdi and Goldwasser [13], a formula on the permanent of the Laplacian matrix of a graph is given as follows.

Lemma 1 (see Lemma 2.1 in [13]). Let $G$ be a graph of order $n$ and $L(G)$ be its Laplacian matrix. Then,

$$
\operatorname{per} L(G)=\sum_{K \subseteq\{1,2, \ldots, n\}} \sum_{H \in H(K)}(-1)^{c_{o}(H)} 2^{c(H)} \prod_{i \notin K} d_{i},
$$

where the first summation is over all induced subgraphs $K$ of $G$, the second summation is over all Sachs spanning subgraph $H$ of $K$, and $c_{o}(H)$ and $c(H)$ denote the number of odd cycles and cycles contained in $H$, respectively.

Combining with Theorem 1 and Lemma 1, we have the following theorem.

Theorem 3. Let $G$ be a bipartite Pfaffian graph of order $n$ and let $\sigma$ be an orientation of $G$ such that $G^{\sigma}$ is a Pfaffian oriented graph. Let $L(G)$ and $L\left(G^{\sigma}\right)$ be the Laplacian matrices of $G$ and $G^{\sigma}$, respectively. Then,

$$
\operatorname{per} L(G)=\operatorname{det} L\left(G^{\sigma}\right) \text {. }
$$

Proof. Let $H$ be any Sachs subgraph of $G$ and the corresponding Sachs oriented subgraph of $G^{\sigma}$ be denoted by $H^{\sigma}$. Since $G$ is bipartite, the order of $H$ is even and $C_{o}(H)=0$. Thus,

$$
(-1)^{c_{o}(H)} 2^{c(H)} \prod_{i \notin H} d_{i}=2^{c(H)} \prod_{i \notin H} d_{i} .
$$
Thus,

$$
(-1)^{c^{+}\left(H^{\sigma}\right)} 2^{c_{e}\left(H^{\sigma}\right)} \prod_{i \notin H^{\sigma}} d_{i}=2^{c_{e}\left(H^{\sigma}\right)} \prod_{i \notin H^{\sigma}} d_{i},
$$

where $c_{e}\left(H^{\sigma}\right)$ denotes the number of all even cycles contained in $H^{\sigma}$. Consequently, the result is as follows.

Theorem 3 is invalid to graphs containing odd cycles. Roughly speaking, any odd cycle has no contribution to the determinant of the Laplacian matrix of an oriented graph. However, for the permanent of the Laplacian matrix of an undirected graph, the effect of odd cycles is completely different. Henceforth, it is difficulty to compute the permanent of the Laplacian matrix of a nonbipartite graph.

In the following, we will show that there exists a class of nonpartite graphs such that the permanent of the Laplacian matrix of those graphs can be formulated as the composite of the determinants of two matrices related to $L(G)$.

Let $\mathbb{G}^{1}$ be the set of all Pfaffian graphs in which each element $G$ of $\mathbb{G}^{1}$ satisfies the following:

(1) Each odd cycle contained in $G$ has length $3(\bmod 4)$

(2) $G$ contains no disjoint odd cycles, that is, two arbitrary odd cycles have at least one common vertex

(3) For any odd cycle $C, G \backslash C$ contains no cycles with length $0(\bmod 4)$

Theorem 4. Let $G$ be a graph with $u \in V(G)$. Then,

$$
\begin{aligned}
\operatorname{per} L(G)= & \operatorname{per} L[G \backslash u] d_{G}(u) \\
& +\sum_{e \text { incident to } u} \operatorname{per} L[G \backslash e]+2 \sum_{u \in C}(-1)^{|C|} \operatorname{per} L[G \backslash C],
\end{aligned}
$$

where the first summation is over all edges incident to $u$ and the second summation is over all cycle $C$ containing the vertex $u$.

Proof. All Sachs subgraphs of $G$ can be divided into three kinds: those that contain the edge $e$, incident to the vertex $u$, as a single edge, those that contain $u$ as a vertex of a cycle, and those that do not. One finds that the sum of all summands of the former is per $\sum_{e} L[G \backslash e]$, the sum of all summands of the second kind is $2 \sum_{u \in C}(-1)^{|C|}$ per $L[G \backslash C]$, and the sum of all summands of the third kind is per $L[G \backslash u] d_{G}(u)$. Thus, the result is as follows.

Theorem 5. Let $G$ be a graph with $e=(u, v) \in E(G)$. Then, $\operatorname{per} L(G)=\operatorname{per} L[G \backslash\{u, v\}]+\operatorname{per} L[G \backslash e]+2 \sum_{e \in C}(-1)^{|C|} \operatorname{per} L[G \backslash C]$,

where the summation is over all cycle $C$ containing the edge e.

Proof. All Sachs subgraphs of $G$ can be divided into three kinds: those that contain the edge $e$ as a single edge, those that contain $e$ as an edge of a cycle, and those that do not. One finds that the sum of all summands of the former is per $L[G \backslash\{u, v\}]$, the sum of all summands of the second kind 
is $2 \sum_{e \in C}(-1)^{|C|}$ per $L[G \backslash C]$, and the sum of all summands of the third kind is per $L[G \backslash e]$. Thus, the result is as follows..

As a consequence of Theorem 5, we have the following result.

Corollary 1. Let $G_{1}$ and $G_{2}$ be two disjoint graphs with $u \in G_{1}$ and $v \in G_{2}$, and $G$ be the graph obtained from $G_{1}$ and $G_{2}$ by adding an edge between $u$ and $v$. Then,

$\operatorname{per} L(G)=\operatorname{per} L\left[G_{1}\right] \operatorname{per} L\left[G_{2}\right]+\operatorname{per} L\left[G_{1} \backslash u\right] \operatorname{per} L\left[G_{2} \backslash v\right]$.

\section{Data Availability}

No data were used to support this study.

\section{Conflicts of Interest}

The authors declare that they have no conflicts of interest regarding the publication of this paper.

\section{Acknowledgments}

This work was supported by Zhejiang Provincial Natural Science Foundation of China (no. LY20A010005) and National Natural Science Foundation of China (nos. 11801512 and 11901525).

\section{References}

[1] F. R. K. Chung, Spectral Graph Theory, CMBS Lecture Notes, American Mathematical Society, vol. 92Providence, RI, , 1997.

[2] B. Mohar, "Some applications of Laplace eigenvalues of graphs, Graph symmetry," in Graph Symmetry, G. Hahn and G. Sabidussi, Eds., Kluwer Academic Publishers, Dordrecht, Netherlands, pp. 225-275, 1997.

[3] R. Merris, K. R. Rebman, and W. Watkins, "Laplacian matrices of graphs: a survey," Linear Algebra and its Applications, vol. 197-198, pp. 143-176, 1994.

[4] R. Merris, “A survey of graph Laplacians," Linear and Multilinear Algebra, vol. 39, no. 1-2, pp. 19-31, 1995.

[5] B. Mohar, "Laplace eigenvalues of graphs: a survey," Discrete Mathematics, vol. 109, no. 1-3, pp. 171-183, 1992.

[6] L. G. Valiant, "The complexity of computing the permanent," Theoretical Computer Science, vol. 8, no. 2, pp. 189-201, 1979.

[7] B. Codenotti, V. Crespi, and G. Resta, "On the permanent of certain $(0,1)$ Toeplitz matrices," Linear Algebra and its Applications, vol. 267, pp. 65-100, 1997.

[8] E. J. Farrell, J. W. Kennedy, and L. V. Quintas, "Permanents and determinants of graphs: a cycle polynomial approach," Journal of Combinatorial Mathematics Combinatorial Computing, vol. 32, pp. 129-137, 2000.

[9] H. Minc, "Bounds for permanents of nonnegative matrices," Proceedings of Edinburg Mathematical Society, vol. 16, pp. 233-237, 1968.

[10] M. Borowiecki, "On spectrum and per-spectrum of graphs," Publications de I'Institut. Mathematique (Beograd), vol. 38, pp. 31-33, 1985.

[11] R. Merris, "The Laplacian permanental polynomial for trees," Czechoslov Mathematics Journal.vol. 32, no. 107, pp. 397-403, 1982.
[12] R. Merris, K. R. Rebman, and W. Watkins, "Permanental polynomials of graphs," Linear Algebra and its Applications, vol. 38, pp. 273-288, 1981.

[13] R. A. Brualdi and J. L. Goldwasser, "Permanent of the laplacian matrix of trees and bipartite graphs," Discrete Mathematics, vol. 48, no. 1, pp. 1-21, 1984.

[14] R. B. Bapat, "A bound for the permanent of the laplacian matrix," Linear Algebra and its Applications, vol. 74, pp. 219-223, 1986.

[15] X. Y. Geng, X. Hu, and S. C. Li, "Further results on permanental bounds for the laplacian matrix of trees," Linear and Multilinear Algebra, vol. 58, no. 5-6, pp. 571-587, 2010.

[16] W. McCuaig, "P ó lya's permanent problem," Electronic Journal of Combinatorics.vol. 11, no. 1, p. 83, 2004.

[17] C. Adiga, R. Balakrishnan, and W. So, "The skew energy of a digraph," Linear Algebra and its Applications, vol. 432, no. 7, pp. 1825-1835, 2010.

[18] S.-C. Gong and G.-H. Xu, "The characteristic polynomial and the matchings polynomial of a weighted oriented graph," Linear Algebra and its Applications, vol. 436, no. 9, pp. 3597-3607, 2012. 